\title{
Accelerated Brain Gray Matter Loss in Fibromyalgia Patients: Premature Aging of the Brain?
}

\author{
Anil Kuchinad, ${ }^{1,2}$ Petra Schweinhardt, ${ }^{1}$ David A. Seminowicz, ${ }^{1}$ Patrick B. Wood, ${ }^{1}$ Boris A. Chizh, ${ }^{4}$ and \\ M. Catherine Bushnell $1,2,3$ \\ ${ }^{1}$ McGill Centre for Research on Pain, ${ }^{2}$ Department of Neurology and Neurosurgery, and ${ }^{3}$ Department of Anesthesia and Faculty of Dentistry, McGill \\ University, Montreal, Quebec, Canada H3A 2B2, and ${ }^{4}$ GlaxoSmithKline, Addenbrooke’s Centre for Clinical Investigation, Addenbrooke's Hospital, \\ Cambridge CB2 2GG, United Kingdom
}

Fibromyalgia is an intractable widespread pain disorder that is most frequently diagnosed in women. It has traditionally been classified as either a musculoskeletal disease or a psychological disorder. Accumulating evidence now suggests that fibromyalgia may be associated with CNS dysfunction. In this study, we investigate anatomical changes in the brain associated with fibromyalgia. Using voxel-based morphometric analysis of magnetic resonance brain images, we examined the brains of 10 female fibromyalgia patients and 10 healthy controls. We found that fibromyalgia patients had significantly less total gray matter volume and showed a 3.3 times greater ageassociated decrease in gray matter than healthy controls. The longer the individuals had had fibromyalgia, the greater the gray matter loss, with each year of fibromyalgia being equivalent to 9.5 times the loss in normal aging. In addition, fibromyalgia patients demonstrated significantly less gray matter density than healthy controls in several brain regions, including the cingulate, insular and medial frontal cortices, and parahippocampal gyri. The neuroanatomical changes that we see in fibromyalgia patients contribute additional evidence of CNS involvement in fibromyalgia. In particular, fibromyalgia appears to be associated with an acceleration of age-related changes in the very substance of the brain. Moreover, the regions in which we demonstrate objective changes may be functionally linked to core features of the disorder including affective disturbances and chronic widespread pain.

Key words: pain; fibromyalgia; functional disorders; voxel-based morphometry; brain anatomy; aging

\section{Introduction}

Fibromyalgia is a disorder of unknown etiology that is characterized by chronic widespread pain and often accompanied by a variety of other symptoms, including sleep impairment, chronic fatigue, affective disturbances and altered stress responses (Wolfe et al., 1990). Although the disorder has been dismissed by many physicians as purely psychological, recent neuroimaging studies show alterations in sensory processing (Gracely et al., 2002) and neurochemical abnormalities (Wood et al., 2006), indicating that fibromyalgia is associated with alterations in the brain's neural functioning.

Changes in brain morphology have been described in chronic pain conditions (Apkarian et al., 2004; Schmidt-Wilcke et al., 2005, 2006), chronic fatigue syndrome (Okada et al., 2004; de Lange et al., 2005), and posttraumatic stress disorder (Villarreal et al., 2002; Corbo et al., 2005; Chen et al., 2006). Because fibromyalgia shares commonalities with these disorders, we hypothesized that fibromyalgia might be associated with neuroanatomical abnormalities as well. Specifically, we sought to determine whether fibromyalgia patients have demonstrable reductions in brain gray matter, particularly in regions involved in pain perception, pain modulation, and stress.

\footnotetext{
Received Jan. 9, 2007; revised Feb. 21, 2007; accepted March 2, 2007

This work was supported by a grant from GlaxoSmithKline.

Correspondence should be addressed to M. Catherine Bushnell, McGill Centre for Research on Pain, 3640 Univer-

sity Street, Room M19, Montreal, Quebec, Canada H3A 2B2. E-mail: Catherine.bushnell@mcgill.ca.

DOI:10.1523/JNEUROSCI.0098-07.2007

Copyright @ 2007 Society for Neuroscience $\quad$ 0270-6474/07/274004-04\$15.00/0
}

\section{Materials and Methods}

Subjects

Ten female fibromyalgia patients (mean age, 52) and 10 healthy female subjects of similar age (mean, 45 years; not significantly different, $t_{(18)}=$ 1.63, $p=0.12$ ) were recruited. Exclusion criteria for both groups included smoking, use of recreational drugs, alcohol consumption of $>3$ U/week, pregnancy, and major medical, neurological, or current psychiatric conditions, including depression (Diagnostic and Statistical Manual of the American Psychiatric Association IV criteria). Patients were not taking antidepressants or anticonvulsants and met the American College of Rheumatology criteria for fibromyalgia (Wolfe et al., 1990). No healthy control subjects met these criteria. The protocol of the study was approved by McGill University Institutional Review Board (Montreal, Quebec, Canada). Written informed consent was obtained from all participants according to the Declaration of Helsinki.

Brain imaging: data acquisition and imaging protocol Imaging was performed using a $1.5 \mathrm{~T}$ Siemens (Erlangen, Germany) Vision scanner with a standard head-coil. Anatomical scans were recorded using a high-resolution T1-weighted anatomical protocol (repetition time, $22 \mathrm{~ms}$; echo time, $9.2 \mathrm{~ms}$; flip angle, $30^{\circ}$; field of view, 256 $\mathrm{mm}$ ). All participants wore earplugs, and their heads were immobilized with a vacuum beanbag pillow, padded earmuffs, and a plastic bar across the bridge of the nose. The voxels in all images before and after preprocessing were $1 \times 1 \times 1 \mathrm{~mm}$.

Voxel-based morphometry

Each image underwent automated correction for intensity nonuniformity and intensity standardization by normalizing gray-level intensities to a common scale (Sled et al., 1998). The corresponding magnetic resonance imagine (MRI) volumes were spatially normalized to a widely 
used T1-weighted MRI template in stereotaxic space, the Montreal Neurological Institute/International Consortium for Brain Mapping (MNI/ ICBM) 152 standard, to adjust for differences in total brain volume and brain orientation. A nonlinear and linear transformation was used to align individual images to the template to preserve cerebral asymmetries (Collins et al., 1994).

Classification of brain tissue into gray matter, white matter, CSF, and background was performed by means of an advanced neural net classifier (Zijdenbos et al., 2002), labeling each voxel based on the MRI signal (see Fig. 2a). Voxels with an effect of insufficient image resolution leading to a mixing of different tissue types were corrected with a partial volume estimation algorithm, which classified a voxel continuously rather than discretely (Kim et al., 2005). Tissue classification was used to extract each tissue type as a binary map; the numbers of voxels in a tissue type provided the total volume of the tissue itself within the binary map. After tissue classification, the skull, brainstem, cerebellum, and dura were removed for additional analysis (Fig. $2 b$ ).

Data smoothing was used to convert the binary data into a range of continuous data and to reduce the effect of individual variation in the exact location of gyri and sulci (Watkins et al., 2001). Images were smoothed with an isotropic Gaussian kernel of $10 \mathrm{~mm}$ full width at half maximum (FWHM) to reduce false positives occurring at smaller smoothing values (Salmond et al., 2002). These smoothed images were used as the three-dimensional maps for gray matter density, measured from the intensity of the image within each voxel.

\section{Statistical analysis}

Comparison of whole-brain tissue volumes between fibromyalgia patients and controls. The general linear model was used to assess differences in total gray matter, white matter and CSF volumes. Because both brain gray and white matter decrease with age (Apkarian et al., 2004; Tisserand et al., 2004), age was included as a covariate. Additionally, multiple linear regression was used to determine the gray matter and total tissue volume percent variance explained by age and time since diagnosis of fibromyalgia. To assess the difference between two regression lines, a difference test between two correlation coefficients was computed, as well as an ANOVA of regression parameters (slopes, Y-intercept, and whether the data are best fit with a common line or each group having its own respective line of best fit; Regress version 1.0, 2000, J. Rochford, McGill University, Montreal, Quebec Canada).

Regional voxel-wise comparisons of gray matter density. Statistical maps of differences in gray matter density between patients and healthy controls at every voxel were obtained using a general linear model (Worsley et al., 1996), using age as a covariate. A groupwise $t$ statistic for each voxel was computed. The number of subjects, data resolution, voxel size, and volume of the search regions were used to compute the critical threshold $t$ value for a two-tailed $\alpha$ significance level of $p<0.05$ after correcting for multiple comparisons, according to the random field theory (Worsley et al., 1996). Because these methods lead to very conservative $t$ values, cluster analyses were also performed, which calculated the probability of observing a group of contiguous voxels with a $t$ value above 3.0, using a smoothing kernel of $10 \mathrm{~mm}$ FWHM. Using these methods, both global searches (with no a priori hypotheses) and directed searches were performed in regions predicted to show anatomical changes. Based on regions shown to have anatomical changes in other voxel-based morphometry studies of chronic pain conditions (Apkarian et al., 2004; Schmidt-Wilcke et al., 2005, 2006) and stress-related disorders, including chronic fatigue syndrome and post-traumatic stress disorder (Villarreal et al., 2002; Okada et al., 2004; Chen et al., 2006), directed searches were performed in anterior, mid, and posterior cingulate cortex, insular cortex, dorsolateral prefrontal cortex, medial prefrontal cortex, and the parahippocampal gyrus.

\section{Results}

As shown in Figure 1a, fibromyalgia patients showed a reduction in gray matter and total brain volume compared with healthy controls. There was no difference in the volume of white matter or CSF between groups. In addition, gray matter volume was negatively correlated with age for fibromyalgia patients, with a
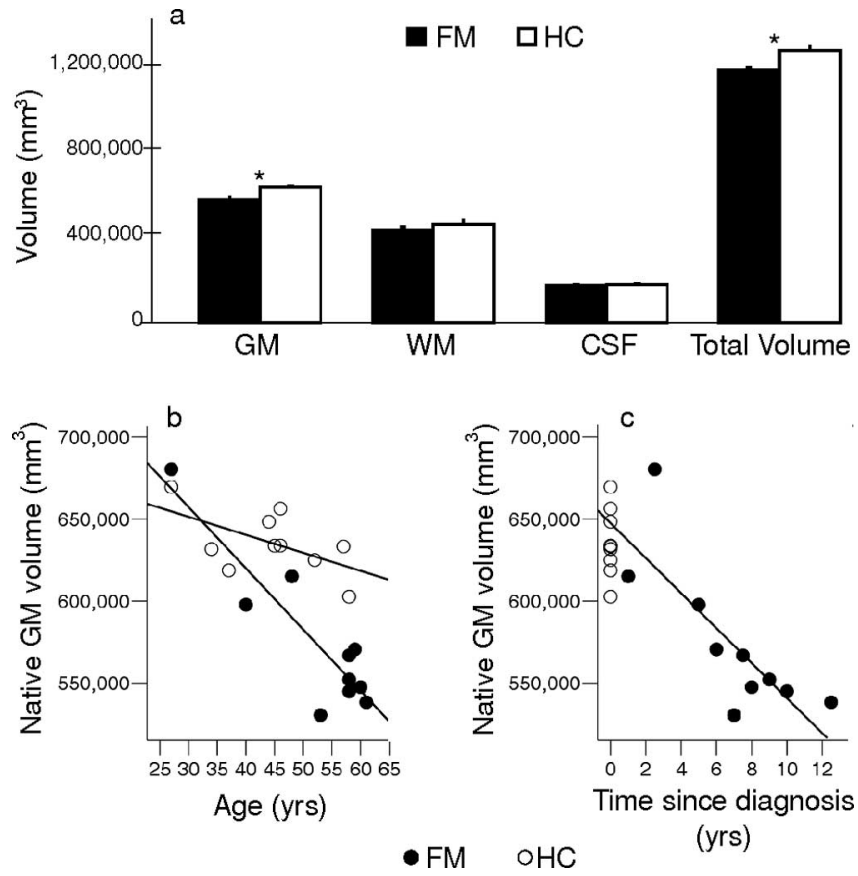

Figure 1. $\boldsymbol{a}$, Brain gray matter, white matter, CSF, and total volume in 10 fibromyalgia patients and 10 healthy control subjects. Values are expressed in millimeters cubed \pm SEM. Including age as a covariate of no interest, fibromyalgia patients had significantly less gray matter and total brain volume than controls. $\boldsymbol{b}$, Brain gray matter tissue volume plotted against age. Gray matter volume correlated significantly with age in fibromyalgia patients $(r=-0.90$; $p<0.001)$, with a similar trend in healthy controls $(r=-0.57 ; p=0.086)$. Fibromyalgia patients showed a significantly steeper age-related decline than controls $\left(F_{(1,18)}=281.57\right.$; $p<0.001)$. c, Gray matter volume versus time since the patient had been diagnosed with fibromyalgia. Gray matter volume was significantly correlated with time since diagnosis $(r=-0.79 ; p=0.007)$, with a loss of $10.5 \mathrm{~cm}^{3}$ in gray matter with each year of diagnosed fibromyalgia. The slope was significantly greater than age-associated loss in healthy controls $\left(F_{(1,18)}=310.78 ; p<0.001\right)$ with a magnitude of decrease equivalent to $\sim 9.5$ years of normal aging seen in healthy controls. Gray matter volume for healthy controls is depicted on the figure, but was not used in the regression analysis. yrs, Years. ${ }^{*} p<0.05$.

similar nonsignificant trend for healthy controls (Fig. 1b). The age-associated loss in gray matter in the fibromyalgia group was $\sim 3.7 \mathrm{~cm}^{3} /$ year, which was significantly greater than the loss in matched controls (only $1.1 \mathrm{~cm}^{3} /$ year). For fibromyalgia patients, three of 10 subjects had normal menstrual cycles and two others were on hormone replacement therapy (HRT); for the healthy controls, seven of 10 were cycling normally and none were on HRT. Nevertheless, Figure $1 b$ shows that the seven fibromyalgia patients over 50 years of age (noncycling) had less gray matter than the three healthy controls in the same age range (also not cycling). For fibromyalgia patients, the gray matter volume was also negatively correlated with time since diagnosis; a loss of 10.5 $\mathrm{cm}^{3}$ in gray matter occurred with each year of diagnosed fibromyalgia (Fig. 1c). As shown in Figure 2 and Table 1, regional voxel-wise comparisons revealed clusters of significantly lower gray matter density in fibromyalgia patients than in healthy controls in the left parahippocampal gyrus, bilateral mid/posterior cingulate gyrus, left insula, and medial frontal cortex. There were no regions with significantly greater gray matter density in fibromyalgia patients compared with healthy controls.

\section{Discussion}

Fibromyalgia patients have abnormal brain gray matter loss These findings reveal for the first time that patients with fibromyalgia have brain gray matter atrophy. Interestingly, the normal 


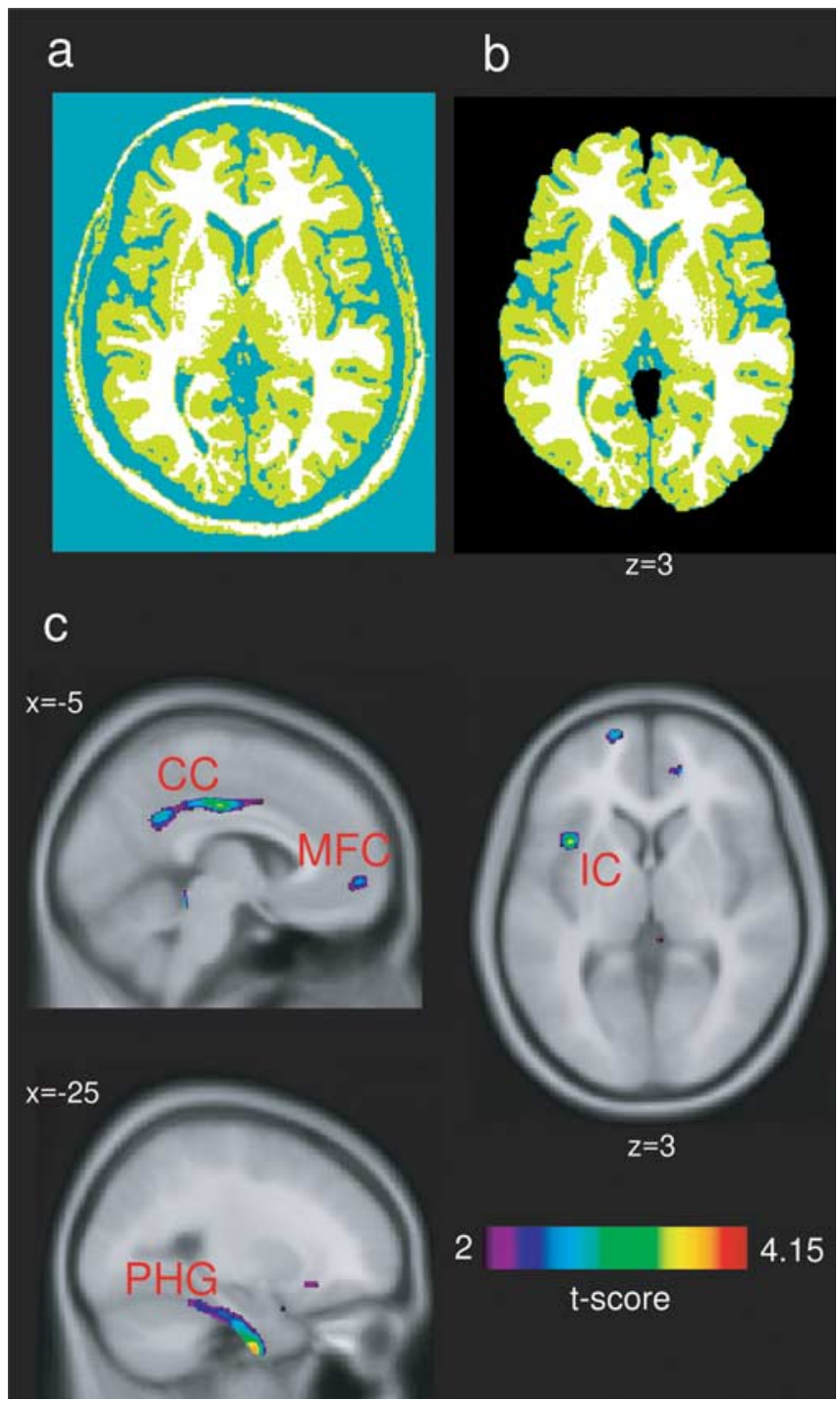

Figure 2. Voxel-wise comparison of gray matter density between fibromyalgia patients and healthy control subjects. In the top panel, an example of the tissue classification procedure on one subject is shown. $\boldsymbol{a}$, The first step of tissue classification into gray matter (green) white matter (white) and CSF (blue). $\boldsymbol{b}$, The same image after applying a mask, which removes the skull, dura, cerebellum, and brainstem. c, Statistical $t$ maps are depicted, with the $t$ threshold set at 2.0 for visualization and superimposed on the MNI/ICBM 152 template. A cluster analysis was used to determine significance levels (for details, see Table 1 and Materials and Methods). Left side of brain is displayed on left. Regions showing significantly less gray matter density for fibromyalgia patients than healthy controls included left parahippocampal gyrus (PHG), left and right mid/posterior cingulate gyrus (CG), left insular cortex (IC), and medial frontal cortex (MFC) (for coordinates, see Table 1).

age-related decrease in gray matter was accelerated in fibromyalgia patients and related to disease duration. The patients, who ranged from 27 to 61 years of age, demonstrated a yearly decrease in gray matter volume more than three times that of age-matched controls. The age-related decrease was even greater than that observed by Resnick et al. (2003) in a much older group of healthy adults (59-85 years), with the fibromyalgia patients in our study showing a decrease of $3.7 \mathrm{~cm}^{3} /$ year and the older healthy subjects showing a decrease of $2.4 \mathrm{~cm}^{3} /$ year. Although menopausal status can clearly have an effect on gray matter volume, the fact that more fibromyalgia patients than healthy controls in our sample were not cycling normally is not likely to account for our findings, because the postmenopausal fibromyalgia patients in our study had less gray matter than the postmenopausal healthy controls. The reduced gray matter was even observed in the two patients who were on HRT, which Erikson et al. (2005) showed increases gray matter volumes in postmenopausal women.

\section{Structural brain changes related to chronic pain and stress disorders}

Accumulating evidence now indicates that a number of chronic pain and stress-related disorders, including chronic low back pain, tension-type headache, chronic fatigue syndrome, and posttraumatic stress disorder, are characterized by gray matter reductions, although the specific regions involved differ among syndromes (Villarreal et al., 2002; Apkarian et al., 2004; Okada et al., 2004; de Lange et al., 2005; Schmidt-Wilcke et al., 2005; Chen et al., 2006). The extensive comorbidity among these disorders suggests that mechanistic similarities may underlie brain atrophy, whereas the regional differences in gray matter decline could explain differences in symptoms.

\section{Mechanisms and implications of gray matter loss}

A possible explanation for the decreased gray matter density in these disorders might be atrophy secondary to excitotoxicity and/or exposure to inflammation-related agents, such as cytokines (Apkarian et al., 2004). It is noteworthy that in fibromyalgia patients, gray matter loss occurred mainly in regions related to stress [parahippocampal gyrus (Herman et al., 2005)] and pain processing [cingulate, insular, and prefrontal cortices (Apkarian et al., 2005)], which might reflect their long-term experience of these symptoms. Because cingulate and prefrontal cortices are particularly implicated in pain modulation (Apkarian et al., 2005) (i.e., inhibition and facilitation of pain), structural changes in these systems could contribute to the maintenance of pain and symptom chronification in fibromyalgia. Furthermore, gray matter atrophy in areas such as parahippocampal and frontal cortices also appears consistent with cognitive deficits characteristic of fibromyalgia (Park et al., 2001). Longitudinal studies are indicated to determine whether the observed structural changes are the cause or the consequence of the disorder. If confirmed, these findings may provide a rationale for exploring neuroprotective approaches in fibromyalgia aimed at symptom treatment or indeed at their reversal.

Table 1. Regions of less gray matter density for fibromyalgia patients than for healthy controls

\begin{tabular}{|c|c|c|c|c|c|c|}
\hline \multirow[b]{2}{*}{ Location } & \multicolumn{3}{|c|}{ Talairach coordinates of peak } & \multirow[b]{2}{*}{ Volume $\left(\mathrm{mm}^{3}\right)$} & \multirow[b]{2}{*}{$p$ value of cluster } & \multirow[b]{2}{*}{ Peak $t$ value } \\
\hline & $x$ & $y$ & $z$ & & & \\
\hline Left parahippocampal gyrus & -25 & -9 & -46 & 3457 & $p<0.001$ & 3.73 \\
\hline Left mid/posterior cingulate gyrus & -14 & -42 & 36 & 2981 & $p<0.001$ & 4.15 \\
\hline Right mid/posterior cingulate gyrus & 10 & -49 & 34 & 1948 & $p<0.001$ & 3.65 \\
\hline Left insular cortex & -38 & 12 & 3 & 515 & $p<0.02$ & 3.48 \\
\hline Medial frontal cortex & 1 & 54 & 22 & 315 & $p=0.05$ & 3.04 \\
\hline
\end{tabular}




\section{References}

Apkarian AV, Sosa Y, Sonty S, Levy RM, Harden RN, Parrish TB, Gitelman DR (2004) Chronic back pain is associated with decreased prefrontal and thalamic gray matter density. J Neurosci 24:10410-10415.

Apkarian AV, Bushnell MC, Treede RD, Zubieta JK (2005) Human brain mechanisms of pain perception and regulation in health and disease. Eur J Pain 9:463-484.

Chen S, Xia W, Li L, Liu J, He Z, Zhang Z, Yan L, Zhang J, Hu D (2006) Gray matter density reduction in the insula in fire survivors with posttraumatic stress disorder: a voxel-based morphometric study. Psychiatry Res 146:65-72.

Collins DL, Neelin P, Peters TM, Evans AC (1994) Automatic 3D intersubject registration of MR volumetric data in standardized Talairach space. J Comput Assist Tomogr 18:192-205.

Corbo V, Clement MH, Armony JL, Pruessner JC, Brunet A (2005) Size versus shape differences: contrasting voxel-based and volumetric analyses of the anterior cingulate cortex in individuals with acute posttraumatic stress disorder. Biol Psychiatry 58:119-124.

de Lange FP, Kalkman JS, Bleijenberg G, Hagoort P, van der Meer JW, Toni I (2005) Gray matter volume reduction in the chronic fatigue syndrome. NeuroImage 26:777-781.

Erickson KI, Colcombe SJ, Raz N, Korol DL, Scalf P, Webb A, Cohen NJ, McAuley E, Kramer AF (2005) Selective sparing of brain tissue in postmenopausal women receiving hormone replacement therapy. Neurobiol Aging 26:1205-1213.

Gracely RH, Petzke F, Wolf JM, Clauw DJ (2002) Functional magnetic resonance imaging evidence of augmented pain processing in fibromyalgia. Arthritis Rheum 46:1333-1343.

Herman JP, Ostrander MM, Mueller NK, Figueiredo H (2005) Limbic system mechanisms of stress regulation: hypothalamo-pituitaryadrenocortical axis. Prog Neuropsychopharmacol Biol Psychiatry 29:1201-1213.

Kim JS, Singh V, Lee JK, Lerch J, Ad-Dab'bagh Y, MacDonald D, Lee JM, Kim SI, Evans AC (2005) Automated 3-D extraction and evaluation of the inner and outer cortical surfaces using a Laplacian map and partial volume effect classification. NeuroImage 27:210-221.

Okada T, Tanaka M, Kuratsune H, Watanabe Y, Sadato N (2004) Mechanisms underlying fatigue: a voxel-based morphometric study of chronic fatigue syndrome. BMC Neurol 4:14.

Park DC, Glass JM, Minear M, Crofford LJ (2001) Cognitive function in fibromyalgia patients. Arthritis Rheum 44:2125-2133.

Resnick SM, Pham DL, Kraut MA, Zonderman AB, Davatzikos C (2003)
Longitudinal magnetic resonance imaging studies of older adults: a shrinking brain. J Neurosci 23:3295-3301.

Salmond CH, Ashburner J, Vargha-Khadem F, Connelly A, Gadian DG, Friston KJ (2002) Distributional assumptions in voxel-based morphometry. NeuroImage 17:1027-1030.

Schmidt-Wilcke T, Leinisch E, Straube A, Kampfe N, Draganski B, Diener HC, Bogdahn U, May A (2005) Gray matter decrease in patients with chronic tension type headache. Neurology 65:1483-1486.

Schmidt-Wilcke T, Leinisch E, Ganssbauer S, Draganski B, Bogdahn U, Altmeppen J, May A (2006) Affective components and intensity of pain correlate with structural differences in gray matter in chronic back pain patients. Pain 125:89-97.

Sled JG, Zijdenbos AP, Evans AC (1998) A nonparametric method for automatic correction of intensity nonuniformity in MRI data. IEEE Trans Med Imaging 17:87-97.

Tisserand DJ, van Boxtel MP, Pruessner JC, Hofman P, Evans AC, Jolles J (2004) A voxel-based morphometric study to determine individual differences in gray matter density associated with age and cognitive change over time. Cereb Cortex 14:966-973.

Villarreal G, Hamilton DA, Petropoulos H, Driscoll I, Rowland LM, Griego JA, Kodituwakku PW, Hart BL, Escalona R, Brooks WM (2002) Reduced hippocampal volume and total white matter volume in posttraumatic stress disorder. Biol Psychiatry 52:119-125.

Watkins KE, Paus T, Lerch JP, Zijdenbos A, Collins DL, Neelin P, Taylor J, Worsley KJ, Evans AC (2001) Structural asymmetries in the human brain: a voxel-based statistical analysis of 142 MRI scans. Cereb Cortex 11:868-877.

Wolfe F, Smythe HA, Yunus MB, Bennett RM, Bombardier C, Goldenberg DL, Tugwell P, Campbell SM, Abeles M, Clark P (1990) The Am College of Rheumatology 1990 criteria for the classification of fibromyalgia. Report of the Multicenter Criteria Committee. Arthritis Rheum 33:160-172.

Wood PB, Patterson II JC, Sunderland JJ, Tainter KH, Glabus MF, Lilien DL (2006) Reduced presynaptic dopamine activity in fibromyalgia syndrome demonstrated with positron emission tomography: a pilot study. J Pain 8:51-58.

Worsley KJ, Marrett S, Neelin P, Vandal AC, Friston KJ, Evans AC (1996) A unified statistical approach for determining significant signals in images of cerebral activation. Hum Brain Mapp 4:58-73.

Zijdenbos AP, Forghani R, Evans AC (2002) Automatic "pipeline" analysis of 3-D MRI data for clinical trials: application to multiple sclerosis. IEEE Trans Med Imaging 21:1280-1291. 